\title{
Detecting Reflection Axes by Energy Minimisation
}

\author{
Dinggang Shen ${ }^{1}$, Horace H. S. Ip ${ }^{2}$ and Eam Khwang Teoh ${ }^{3}$, \\ ${ }^{1}$ Department of Radiology, Johns Hopkins University, USA \\ ${ }^{2}$ Image Computing Group, Department of Computer Science, City University of Hong Kong, Hong Kong \\ ${ }^{3}$ School of Electrical and Electronic Engineering, Nanyang Technological University, Singapore \\ Email: dgshen@cbmv.jhu.edu, cship@cityu.edu.hk, eekteoh@ntu.edu.sg
}

\begin{abstract}
In this paper, we formulated the problem of detecting reflectional symmetries as the problem of minimising an asymmetric energy term. The local minima of the asymmetric energy correspond to the reflectional symmetric axes of an object. Typical experiments show the correctness and the robustness of the proposed technique.
\end{abstract}

\section{Introduction}

Reflectional symmetry is a very important type of symmetries in planar image and the presence of such cue helps in performing a wide variety of tasks such as modelbased object matching and object recognition [1,2,12]. Various methods have been proposed to detect the reflection-symmetric axes of an object. However, the review given below shows that none of the previously proposed methods are complete in the sense that they are unable to detect all reflection-symmetric axes from every object.

Some methods focused on detecting one reflectionsymmetric axis. Labonte et al [3] studied the problem of detecting global bilateral symmetry in images consisting of dense arrangements of local features, such as dots or oriented segments. Zielke et al [4] only looked at vertical or near-vertical symmetric axes in an image for carfollowing.

Other methods attempted to detect more than one reflection-symmetric axes. Atallah [5] detected the axes of reflectional symmetries by first determining the centroid position and then using a string pattern matching technique. However, it is only applicable to planar figures made up of (possibly intersecting) segments, circles, points, etc. Marloa [6] presented an algorithm that required the evaluation of some rational functions. Masuda et al [7] detected the reflectional symmetry by performing correlation with the reflected images. Its disadvantage is high computational cost and memory requirement. Sun and Sherrah [8] formulated the symmetry detection problem as a correlation of the Gaussian image. However, this technique is unable to determine whether the input image is symmetric or not.

In this paper, we define a novel asymmetric energy function, based on a set of Generalised Complex (GC) $[10,11]$ moments computed for an object. The necessary and sufficient condition for the object invariant under a reflection about the $x$-axis is that, the asymmetric energy must be close to zero. We determined the local minima of the asymmetric energy by using the steepest descent technique, where the initial positions are determined by the most important term (with the largest magnitude) in the asymmetric energy function. Since not all the local minima correspond to the negative angles of the reflection-symmetric axes, two criteria are thus proposed to screen out the false angles.

The advantages of our method are: (a) all the global reflection-symmetric axes can be detected, and (b) the detection is accurate, since the steepest descent method is used to locate and fine tune the angles of the reflectionsymmetric axes.

\section{Properties of reflection-symmetric object}

In this section, we analyse the properties related to the GC moments (Theorem 2.1) and the novel asymmetric energy (Theorem 2.2) of the reflection-symmetric object.

The definition of GC moment for any image is given in definition 2.1, where $f(r, 6)$ is a function of a centred image in the polar coordinate.

Definition 2.1 The $p q$-order generalised complex (GC) moment of image function $f(r, 6)$ is defined as:

$$
G C_{p, q}=R_{p, q} e^{j \varphi_{p, q}}=\frac{1}{2 \pi} \int_{0}^{2 \pi} \int_{0}^{\infty} f(r, \theta)\left(r^{p} e^{j q \theta}\right) r d r d \theta
$$

where $p$ is a non-negative integer, and $q$ a positive integer.

An image is said to be reflection-symmetric, if it is invariant to reflection with respect to one or more straight lines. Mathematically, the reflection-symmetric object satisfies: 


$$
f(r, \phi+6)=f(r, \phi-6)
$$

where $\phi$ is an angle from the $x$-axis to the symmetric axis. Note that this mathematical form is also a necessary and sufficient condition for an object $f(r, 6)$ to be reflectionsymmetric about the $x$-axis [9]. From this property, we can infer new property in Theorem 2.1 .

Theorem 2.1 All GC moments $G C_{p q}$ are real, if the input image object $f(r, 6)$ is invariant under a reflection about the $x$-axis.

In practice, we have to determine the reflectionsymmetric axes of an object by a limited number of GC moments. Accordingly, the order $p$ in $G C_{p, q}$ is usually fixed at a particular value $P$ when the GC moments are used for detecting reflection axes $[10,11]$. If we use only $N$ GC moments, $\left\{G C_{P . q} \mid 0 \leq q \leq(N-1)\right\}$, to detect the reflection-symmetric axes. Then, we can define a total energy and an asymmetric energy by these $N$ GC moments:

$$
\begin{gathered}
E_{\text {Toral }}=2 \pi \sum_{q=0}^{N-1}\left\|G C_{P q}\right\|^{2}=2 \pi \sum_{q=0}^{N-1} R_{P q}^{2}, \\
E_{\text {Asym }}=2 \pi \sum_{q=0}^{N-1}\left(R_{P, q} \sin \left(\varphi_{P, q}\right)\right)^{2} .
\end{gathered}
$$

From Theorem 2.1, we can easily obtain Theorem 2.2.

Theorem 2.2 The asymmetric energy of an object $f(r, 6)$ must be zero, i.e. $E_{\text {Asym }}=2 \pi \sum_{q=0}^{N-1}\left(R_{P . q} \sin \left(\varphi_{P . q}\right)\right)^{2}=0$, if it is invariant under a reflection about $x$-axis.

Given these two theorems, we can then derive an energy-based reflection symmetry detection algorithm.

\section{Minimisation of the asymmetric energy}

In this section, we describe the joint process which determines, (a) whether an object $f(r, 6)$ is reflectionsymmetric or not, and (b) the location of all the reflectionsymmetric axes.

In the following, we will analyse the variation of the asymmetric energy with the rotation angle $\alpha$ of the reflection-symmetric axis.

Let the image object $f(r, 6)$ be counterclockwise rotated by an angle of $\alpha$, and become $f(r, 6-\alpha)$. Then the corresponding $\mathrm{GC}$ moments of the rotated image object $f(r, 6-\alpha)$ become $G C_{P, q} \cdot e^{j q \alpha}$. Similarly, the asymmetric energy $\left(E_{A s y m}^{\alpha}\right)$ of the rotated image object $f(r, 6-\alpha)$ becomes: $E_{\text {Asym }}^{\alpha}=2 \pi \sum_{q=0}^{N-1}\left(R_{P_{. q}} \sin \left(\varphi_{P_{. q}}+q \alpha\right)\right)^{2}$.

Hence, the problems of determining (a) whether an object is reflection-symmetric and (b) the locations of all its reflection-symmetric axes become the problem of finding the angle set $\left\{\alpha_{i} \mid 1 \leq i \leq M\right\}$ which makes $E_{\text {Asym }}^{\alpha_{i}}$ equal to zero, i.e. $E_{A s y m}^{\alpha_{i}}=0$. If the number $M$ is not equal to zero, then the object $f(r, 6)$ has $M$ reflectionsymmetric axes that pass through the origin at the angles $\left\{-\alpha_{i} \mid 1 \leq i \leq M\right\}$. (Notice here that the angles for the reflection-symmetric axes of the object $f(r, 6)$ are $-\alpha_{i}$, not $\alpha_{i}$.) On the other hand, if $M=0$, then the object is not reflection-symmetric.

Owing to the facts that noise exists in real applications and also the object might not be perfectly reflectionsymmetric, there might not exist an angle $\alpha$ exactly making $E_{\text {Asym }}^{\alpha_{i}}$ zero. Therefore, in practice, we need to first obtain the angles $\alpha_{i}$ with the local minima of $E_{A s y m}^{\alpha_{i}}$, and then use certain criteria to determine whether the axes that pass through the origin at angles $-\alpha_{i}$ are indeed the true reflection-symmetric axes.

The local minima of the asymmetric energy can be located using the steepest descent method (gradient-based iteration method). The initialisation of steepest descent method is provided as follows.

Let's assume that $R_{P, Q}$, where $0 \leq Q \leq(N-1)$, be the largest value in the set of all $N$ magnitudes $\left\{R_{P . q} \mid 0 \leq q \leq(N-1)\right\}$. Therefore, we can say that, the term $2 \pi\left(R_{P, Q} \sin \left(\varphi_{P, Q}+Q \alpha\right)\right)^{2}$ in $E_{A s y m}^{\alpha}$ is the dominant term affecting the value of $E_{\text {Asym }}^{\alpha}$. Also, the potential local minima should be near to the angles that make this dominant term $2 \pi\left(R_{P, Q} \sin \left(\varphi_{P, Q}+Q \alpha\right)\right)^{2}$ zero. We can easily derive the angles satisfying the requirement,

$$
\begin{aligned}
& 2 \pi\left(R_{P, Q} \sin \left(\varphi_{P, Q}+Q \alpha\right)\right)^{2}=0, \text { i.e. } \\
& \alpha_{i}=-\frac{\varphi_{P, Q}+i \pi}{Q}, i=0,1, \ldots,(Q-1) .
\end{aligned}
$$

Thus the evaluated angles $\alpha_{i}$ can be used as the initial variables $\alpha_{i}^{(0)}=\alpha_{i}$ of the steepest descent method. More accurate (or refined) angles can then be obtained by the following iteration equation.

$$
\alpha_{i}^{(t+1)}=\alpha_{i}^{(t)}-\left.\eta \frac{\partial E_{\text {Asym }}^{\alpha}}{\partial \alpha}\right|_{\alpha=a_{i}^{(i)}}, i=0,1, \ldots,(Q-1),
$$

where $\eta$ is the learning rate and $\frac{\partial E_{A s y m}^{\alpha}}{\partial \alpha}$ is the derivative with respect to $\alpha$, given in details as follows:

$$
\frac{\partial E_{A s v m}^{\alpha}}{\partial \alpha}=2 \pi \sum_{q=0}^{N-1}\left(R_{P, q}\right)^{2} \cdot \sin \left(2 \varphi_{P, q}+2 q \alpha\right) \cdot q .
$$

After convergence, we can then get $Q$ final angles $\alpha_{i}^{\left(T_{i}\right)}$, where $T_{i}$ is the steps used to make $\alpha_{i}^{(\ell)}$ converged. However, not all such convergent angles exactly 
correspond to the reflection-symmetric axes of the object, we thus suggest two criteria for selecting the true ones from the set of convergent angles $\alpha_{i}^{\left(T_{i}\right)}$. Two criteria suggested here are as follows:

(a) $E_{\text {Asym }}^{\alpha} / E_{\text {Total }} \leq \varepsilon_{1}$, and (b) $\left\|\alpha_{i}^{\left(T_{i}\right)}-\alpha_{i}^{(0)}\right\| \leq \varepsilon_{2}$.

The criterion (a) means that $E_{\text {Asym }}^{\alpha}$ should only be a small portion of the total energy $E_{\text {Total }}$, since Theorem 2.2 requires that the asymmetric energy be zero for any reflection-symmetric object $f(r, 6)$. Criterion (b) indicates that the convergent angle $\alpha_{i}^{\left(T_{i}\right)}$ should be very close to the initial angle $\alpha_{i}^{(0)}$, otherwise, the term $\left.2 \pi\left(R_{P, Q} \sin \left(\varphi_{P, Q}+Q \alpha\right)\right)^{2}\right|_{\alpha=\alpha_{i}^{T_{i}}} \quad$ will become large and $E_{\text {Asym }}^{\alpha}$ will be large too.

The complete algorithm for detecting reflectionsymmetric axes of the object is summarised as:

(1) Use a fixed order $p=P$ and calculate the first $N$ GC moments of an image, i.e. $\left\{G C_{P, q} \mid 0 \leq q \leq(N-1)\right\}$.

(2) Select the largest magnitude $R_{P, Q}$, where $0 \leq Q \leq(N-1)$, from the set of the $N$ magnitudes $\left\{R_{P, q} \mid 0 \leq q \leq(N-1)\right\}$.

(3) Obtain the angles $\alpha_{i}=-\frac{\varphi_{P, Q}+i \pi}{Q}, i=0,1, \ldots,(Q-1)$, that satisfy the equation $2 \pi\left(R_{P, Q} \sin \left(\varphi_{P, Q}+Q \alpha\right)\right)^{p}=0$.

(4) Regard the angles $\alpha_{i}, i=0,1, \ldots,(Q-1)$, as the initial variables and use the steepest descent method to achieve the convergent variables.

(5) Apply the two constraints suggested in the last section to screen out the false reflection-symmetric angles from the set of the convergent angles. These two constraints are (a) $E_{\text {Asym }}^{\alpha} / E_{\text {Total }} \leq \varepsilon_{1}$, and (b) $\left\|\alpha_{i}^{\left(T_{i}\right)}-\alpha_{i}^{(0)}\right\| \leq \varepsilon_{2}$.

(6) The real reflection-symmetric axes are those which pass through the origin at angles $-\alpha_{i}^{\left(T_{i}\right)}$ and satisfy the constraints in step (5).

\section{Experiments and conclusion}

To clearly demonstrate the ability and procedure of our energy-based method, two typical objects in figures 1 are used as examples. Grey thick lines in figures 1 denote the detected reflection-symmetric axes using the energybased method, while the black thin lines show the potential axes as detected by only using the GC moment with the largest magnitude.

For the object in figure 1(a), its true number of reflection-symmetric axes is less than that determined by the most dominant $\mathrm{GC}$ moment $\left(G C_{0,4}\right)$ of this object. For the object in figure 1(b), our energy-based method correctly detects that no real reflection-symmetric axis actually exists, but three false axes were determined by the object's most dominant GC moment $G C_{0,3}$.

In figures 2 and 3 , the black curve represents the asymmetric energy $E_{A s y m}^{\alpha}$ in the domain $0 \leq \alpha \leq \pi$, while the grey curve represents the variation of the term $2 \pi\left(R_{P, Q} \sin \left(\varphi_{P, Q}+Q \alpha\right)\right)^{2}$ with the angle $\alpha$. The dotted vertical lines represent the initial angles as calculated from the most dominant GC moment of the object, while the solid vertical lines represent the final angle positions converged by the steepest descent method.

- Experiment on the object in figure 1(a)

Among the $N=15 \mathrm{GC}$ moments of the object in figure 1(a), the magnitude of $G C_{0,4}$ is the largest. The reflectionsymmetric axes as determined by only $G C_{0,4}$ is shown in figure 1(a1). Table 1 shows the corresponding angle $\alpha_{i}$, the initial angles $\alpha_{i}^{(0)}=\alpha_{i}$, and the convergent angles $\alpha_{i}^{\left(T_{i}\right)}$. Here $i=0,1, \ldots, 3$. In figure 2 , the grey curve represents the term $2 \pi\left(R_{0.4} \sin \left(\varphi_{0.4}+4 \alpha\right)\right)^{2}=E$, and the dotted vertical lines represent the initial angels calculated by $G C_{0,4} .54$ iterations were needed to obtain all four local minima. Figure 2 visually shows that three out of the four detected angles cannot be the true reflection axes, since their corresponding $E_{\text {Asym }}^{\alpha_{i}}$ are too large and hence unable to meet our first constraint. Figure 1(a2) therefore shows a single reflection-symmetric axis with the angle -0.304 detected for this object.

\section{- Experiment on the object in figure 1(b)}

For this object, the magnitude of $G C_{0,3}$ is the largest one among all the 15 magnitudes. The reflectionsymmetric axes as determined by $G C_{0,3}$ are shown in figure 1(b1), all of which are actually the false axes. The corresponding angle $\alpha_{i}$, the initial angles $\alpha_{i}^{(0)}=\alpha_{i}$, and the convergent angles $\alpha_{i}^{\left(T_{i}\right)}$ are shown in Table 2. Here, $i=0,1,2$. In figure 3 , the grey curve represents the term $2 \pi\left(R_{0.3} \sin \left(\varphi_{0.3}+3 \alpha\right)\right)^{2}=E$, and the dotted vertical lines represent the initial angels calculated by $G C_{0,3} .29$ iterations were required to obtain all three local minima. The figure 3 visually shows that none of these angles can be the true reflection axes, since both their $E_{\text {Asym }}^{\alpha_{i}}$ and $\left\|\alpha_{i}^{\left(T_{i}\right)}-\alpha_{i}^{(0)}\right\|$ are too large, and unable to satisfy our two constraints. So in figure $1(\mathrm{~b} 2)$, no reflection-symmetric axis is detected. 
In this paper, we formulated the problem of detecting reflection symmetries as the problem of minimising the asymmetric energy. Theoretical and experimental results have shown the correctness and robustness of the proposed technique.

\section{Acknowledgement}

This work is part-supported by a HKSAR Research grant Council, CERG grant No. 9040437.

\section{References}

1. W. G. Oh, M. Asada, and S. Tsuji, "Model-based matching using skewed symmetry information", Proc. Int'l Conf. Pattern Recognition, pp.1043-1045, 1988.

2. G. Marola, "Using symmetry for detecting and locating objects in a picture", Comput. Vision Graphics Image Process, 46, pp.179-195, 1989.

3. F. Labonte, Y. Shapira, and P. Cohen, "A perceptually plausible model for global symmetry detection, Proceedings", Fourth International Conference on Computer Vision, pp.258-263, 1993.

4. T. Zielke, M. Brauckmann and W. V. Seelen, "Intensity and edge-based symmetry detection with an application to carfollowing", Computer Vision, Graphics, and Image Processing: Image Understanding, Vol.58, No.2, pp.177-190.

5. M. Atallah, "On symmetry detection", IEEE Trans. on computer, Vol.34, No.7, 1985.

6. G. Marola, "On the detection of the axes of symmetry of symmetric and almost symmetric planar images", IEEE Trans. on Pattern Analysis and Machine Intelligence, Vo.11, No.1, pp.104-108, 1989.

Table 1 Angles calculated for the object in figure 1(a)

\begin{tabular}{|l|c|c|c|c|}
\hline \multicolumn{5}{|c|}{$G C_{P, Q}=G C_{0.4}=0.3471 e^{j 1.9672}, T_{i}=54$} \\
\hline Angles & ang 1 & ang 2 & ang 3 & ang 4 \\
\hline$\alpha_{i}$ & -0.491 & -1.277 & -2.062 & -2.848 \\
\hline Ini. ang. $\alpha_{i}^{(0)}$ & -0.491 & -1.277 & -2.062 & -2.848 \\
\hline Converg. ang. $\alpha_{i}^{\left(T_{i}\right)}$ & -0.685 & -1.278 & -2.160 & -2.837 \\
\hline Final ang. & & & & $\sqrt{ }$ \\
\hline
\end{tabular}

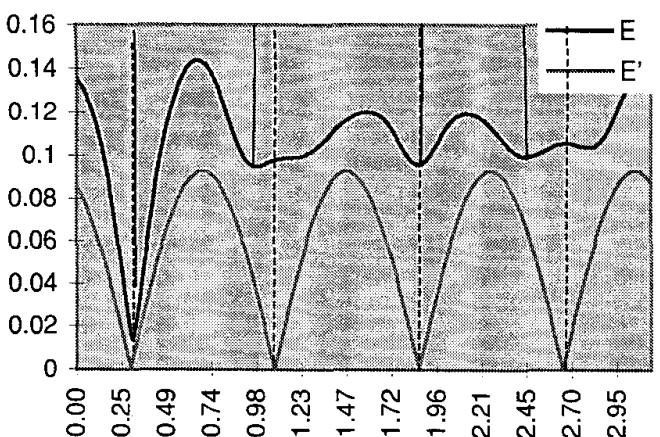

Fig. 2 The local minima calculated in asymmetric energy $E_{A s y m}^{\alpha}=E$. Here $2 \pi\left(R_{0,4} \sin \left(\varphi_{0,4}+4 \alpha\right)\right)^{2}=E^{\prime}$. Solid vertical lines denote the convergent angles, while dotted vertical lines denote the initial angles.
7. T. Masuda, K. Yamamoto and H. Yamada, "Detection of partial symmetry using correlation with rotated-reflected images", Pattern Recognition, Vol.26, No.8, 1993.

8. C. Sun and J. Sherrah, "3D symmetry detection using the extended Guassian image", IEEE Trans. on Pattern Analysis and Machine Intelligence, Vol.19, No.2, pp.164-168, 1997.

9. Dinggang Shen, Horace H. S. Ip, Kent K T Cheung, and Eam Khwang Teoh, "Symmetry detection by generalized complex (GC) moments: a close-form solution", IEEE Trans. on PAMI, 21(5):466-476, May 1999.

10. Dinggang Shen, Horace H. S. Ip, "Generalized affine invariant image normalization", IEEE Trans. on PAMI, 19(5):431-440, May 1997.

11. Dinggang Shen, Horace H. S. Ip, "Optimal axes for defining the orientations of shapes", IEE Electronic Letters, 32(20), 1873-1874, Sept. 1996.

12. H. Zabrodsky, S. Peleg, and D. Avnir, "Symmetry as a continuous feature", IEEE Trans. on PAMI, 17(12): 1154 1166, Dec 1995.

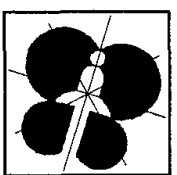

(a1)

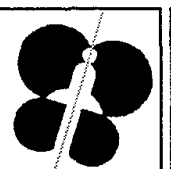

(a2)

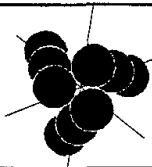

(b1)

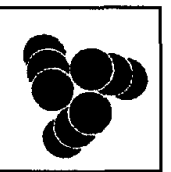

(b2)
Fig. 1 Reflection symmetries detection on two objects. In subfigures (a1) and (b1), those axes are determined by only the most dominant $\mathrm{GC}$ moment $G C_{P, Q}$. While in subfigures (a2) and (b2), those reflection symmetries are determined by our energy-based method which considering all the NGC moments.

Table 2 Angles calculated for the object in figure 1(b)

\begin{tabular}{|l|c|c|c|}
\hline \multicolumn{4}{|c|}{$G C_{P, Q}=G C_{0,3}=0.3515 e^{-0.4857 j}, T_{i}=29$} \\
\hline Angles & ang 1 & ang 2 & ang 3 \\
\hline$\alpha_{i}$ & 0.162 & -0.885 & -1.932 \\
\hline ini. ang. $\alpha_{i}^{(0)}$ & 0.162 & -0.885 & -1.932 \\
\hline converg. ang. $\alpha_{i}^{\left(T_{i}\right)}$ & 0.236 & -0.812 & -1.858 \\
\hline final ang. & & & \\
\hline
\end{tabular}

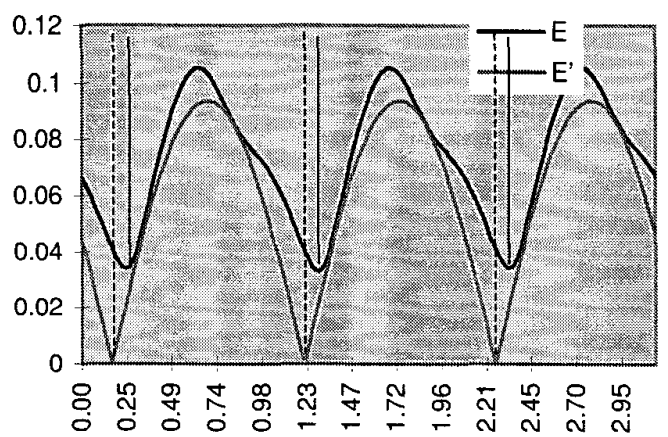

Fig. 3 The local minima calculated in asymmetric energy $E_{A s y m}^{\alpha}=E$. Here $2 \pi\left(R_{0,3} \sin \left(\varphi_{0,3}+3 \alpha\right)\right)^{2}=E$ '. Solid vertical lines denote the convergent angles, while dotted vertical lines denote the initial angles. 